\title{
Modification of the Gay-Berne potential for improved accuracy and speed
}

Rasmus A. X. Persson, 1 a

Department of Chemistry \& Molecular Biology, University of Gothenburg, SE-412 96 Göteborg, Sweden

A modification of the Gay-Berne potential is proposed which is about $10 \%$ to $20 \%$ more speed efficient (that is, the original potential runs $15 \%$ to $25 \%$ slower, depending on architecture) and statistically more accurate in reproducing the energy of interaction of two linear Lennard-Jones tetratomics when averaged over all orientations. For the special cases of end-to-end and side-by-side configurations, the new potential is equivalent to the Gay-Berne one.

a) Electronic mail: rasmus.persson@chem.gu.se 
The Gay-Berne potential ${ }^{1}$ is a coarse-grained potential designed specifically to reproduce the energy of interaction between two linear, tetratomic Lennard-Jones molecules. As such, it allows the approximate treatment of a linear, rigid polyatomic segment by use of a single interaction site, reducing the amount of looping - and hence the calculational effort - drastically when calculating energies, forces and torques in molecular mechanics. The Gay-Berne potential is not the only single-site potential for linear molecules or molecular moieties lacking substantial electric multipole momoments, 2 - 9 but it is arguably the most widely used. In this Note, we present a variation of the Gay-Berne potential which undeniably improves even further on the computational economy and also on the accuracy. For ease of reference and of comparison, the original functions are also reproduced.

The Gay-Berne potential, for intermolecular distance $r$, is given in eq. (1).

$$
V\left(\widehat{u}_{1}, \widehat{u}_{2}, \widehat{r}\right)=\epsilon\left(\widehat{u}_{1}, \widehat{u}_{2}, \widehat{r}\right)\left[\left(\frac{\sigma_{0}}{r-\sigma\left(\widehat{u}_{1}, \widehat{u}_{2}, \widehat{r}\right)+\sigma_{0}}\right)^{12}-\left(\frac{\sigma_{0}}{r-\sigma\left(\widehat{u}_{1}, \widehat{u}_{2}, \widehat{r}\right)+\sigma_{0}}\right)^{6}\right] .
$$

In this equation the $\epsilon\left(\widehat{u}_{1}, \widehat{u}_{2}, \widehat{r}\right)$ and $\sigma\left(\widehat{u}_{1}, \widehat{u}_{2}, \widehat{r}\right)$ are auxiliary functions that depend both on the molecular orientations, expressed through the unit vectors $\widehat{u}_{1}$ and $\widehat{u}_{2}$, and on the orientation of the intermolecular vector, expressed by $\widehat{r}$. Gay and Berne ${ }^{1}$ provide the following forms for these functions:

$$
\begin{aligned}
& \sigma\left(\widehat{u}_{1}, \widehat{u}_{2}, \widehat{r}\right)=\sigma_{0}\left(1-\frac{\chi}{2}\left\{\frac{\left(\widehat{r} \cdot \widehat{u}_{1}+\widehat{r} \cdot \widehat{u}_{2}\right)^{2}}{1+\chi\left(\widehat{u}_{1} \cdot \widehat{u}_{2}\right)}+\frac{\left(\widehat{r} \cdot \widehat{u}_{1}-\widehat{r} \cdot \widehat{u}_{2}\right)^{2}}{1-\chi\left(\widehat{u}_{1} \cdot \widehat{u}_{2}\right)}\right\}\right)^{-\frac{1}{2}} \\
& \epsilon\left(\widehat{u}_{1}, \widehat{u}_{2}, \widehat{r}\right)=\epsilon_{0}\left[1-\chi^{2}\left(\widehat{u}_{1} \cdot \widehat{u}_{2}\right)^{2}\right]^{-\frac{1}{2}}\left\{1-\frac{\chi^{\prime}}{2}\left[\frac{\left(\widehat{r} \cdot \widehat{u}_{1}+\widehat{r} \cdot \widehat{u}_{2}\right)^{2}}{1+\chi^{\prime}\left(\widehat{u}_{1} \cdot \widehat{u}_{2}\right)}+\frac{\left(\widehat{r} \cdot \widehat{u}_{1}-\widehat{r} \cdot \widehat{u}_{2}\right)^{2}}{1-\chi^{\prime}\left(\widehat{u}_{1} \cdot \widehat{u}_{2}\right)}\right]\right\}^{2}
\end{aligned}
$$

where $\sigma_{0}$ and $\epsilon_{0}$ are constants, and $\chi$ and $\chi^{\prime}$ relate to the anisotropy of the interaction and are defined by $\chi=\left(l^{2}-1\right) /\left(l^{2}+1\right)$ and $\chi^{\prime}=(\sqrt{d}-1) /(\sqrt{d}+1)$, where $l$ is the steric length-to-breadth ratio (the axial ratio) and $d$ is the ratio of the binding energy in the sideby-side configuration to that of the end-to-end configuration. A comparison of the energy of interaction between the model system it was designed to replicate, and the Gay-Berne potential itself is shown in Figure 1, using the original parameter set advocated by Gay and Berne themselves. We shall keep the general form of eq. (1), but replace the auxiliary functions $\sigma$ and $\epsilon$ by more efficient alternatives.

The choices for $\sigma$ and $\epsilon$ that we make are inspired by Ref. 10, in which the equipotential surface of the Lennard-Jones potential was redefined to that of an ellipsoid. There, the philosophy of Berne and Pechukas ${ }^{6}$ and of Kihara ${ }^{3}$ - that the anisotropic intermolecular 


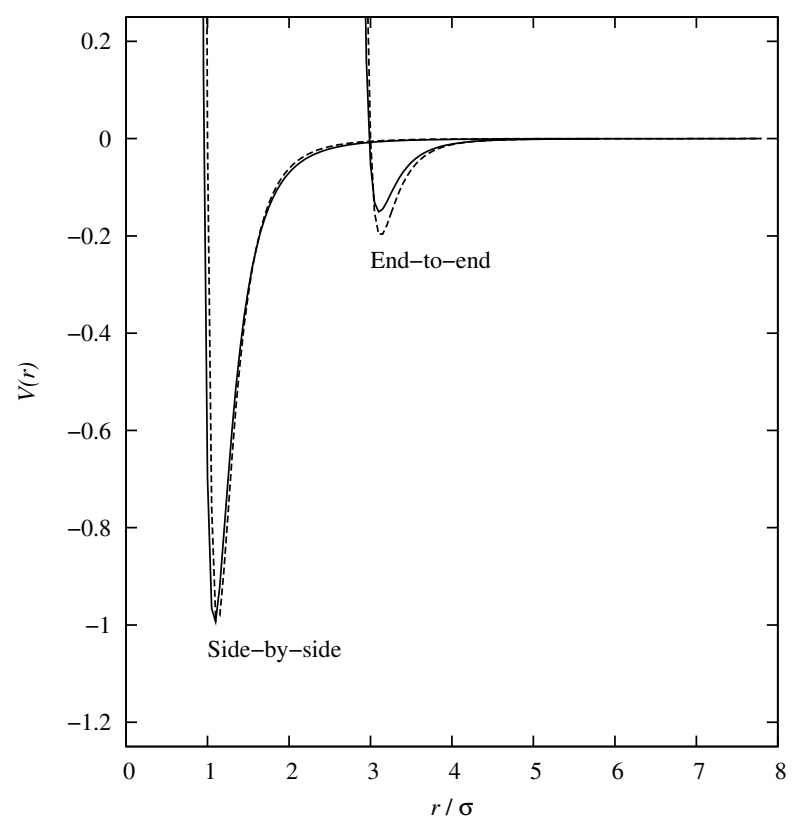

FIG. 1. Energy of interaction of two linear, tetratomic Lennard-Jones molecules of bond length $2 \sigma / 3$ (full line) and their Gay-Berne approximation (dashed line) with $l=3$ and $d=5$. The binding energy of the side-by-side configuration is set to unity in both cases. The unit of length is $\sigma$ of the Lennard-Jones interaction.

potential is obtained by replacing the distance scale by an anisotropic function - was strictly adhered to. In abandoning that principle for the prescription of Gay and Berne ${ }^{1}$ so that we displace rather than dilate the interaction potential, using the anisotropic $\sigma$ function of Ref. 10 and its close analog for the $\epsilon$ function in eq (1), we arrive at the modified Gay-Berne potential. To avoid a notational cluttering and as confusion is unlikely to arise, we do not distinguish these new functions through notation from their Gay-Berne counterparts. The new angular functions are represented by

$$
\begin{aligned}
\sigma\left(\widehat{u}_{1}, \widehat{u}_{2}, \widehat{r}\right) & =\sigma_{0}\left[1+\frac{l-1}{2}\left(\left|\widehat{r} \cdot \widehat{u}_{1}\right|+\left|\widehat{r} \cdot \widehat{u}_{2}\right|\right)\right] \\
\epsilon\left(\widehat{u}_{1}, \widehat{u}_{2}, \widehat{r}\right) & =\epsilon_{0}\left[1+\frac{d^{-1}-1}{2}\left(\left|\widehat{r} \cdot \widehat{u}_{1}\right|+\left|\widehat{r} \cdot \widehat{u}_{2}\right|\right)\right]
\end{aligned}
$$

These new functions give the same dashed curves in Figure 1 as the original ones. Important as such agreement might be, those two geometries only represent a subset of all possibilities; a more thorough test of the model is to calculate its average error over all possible configurations. The average absolute error, defined by $\left\langle\left|E_{\text {exact }} / E_{\text {model }}-1\right|\right\rangle$ where the angle brackets denote an unweighted angular average, is shown in Table $\mathrm{I}$ for both the original 
and the modified Gay-Berne potential as a function of intermolecular separation. $E_{\text {exact }}$ is the energy of the dimer of the linear tetratomic, as used in the original paper, 1 and $E_{\text {model }}$ is the energy of interaction of the Gay-Berne potential, original or modified, in the same geometry. Both energies are normalized by their respective side-by-side binding energy. It is worth pointing out that this comparison is made with the original Gay-Berne parameter set, optimized for this situation, and that further improvement is perhaps possible with adjustments. It is a chief strength of the modification that it does not need reparametrization to improve upon the original.

TABLE I. Angular-averaged unsigned errors for selected intermolecular distances $r$ for the original and modified Gay-Berne potential with respect to the linear Lennard-Jones tetratomic, obtained by unbiased Monte Carlo sampling over $10^{8}$ cycles. Identical values of $l=3$ and $d=5$ are used in the comparison.

\begin{tabular}{lrr}
\hline \hline$r / \sigma$ & Error, original & Error, modified \\
\hline 1.5 & 1300000 & 5.2 \\
2.0 & 2.9 & 1.1 \\
2.5 & 2.0 & 0.96 \\
3.0 & 1.2 & 0.72 \\
3.5 & 1.3 & 0.62 \\
4.0 & 1.3 & 0.51 \\
4.5 & 1.4 & 0.41 \\
5.0 & 1.4 & 0.33 \\
5.5 & 1.4 & 0.35 \\
6.0 & 1.5 & 0.47 \\
\hline \hline
\end{tabular}

One further virtue of these new angular functions is the ease with which they can be extended to cover also the case of the interaction between non-identical elongations and binding energies. The natural generalization of the anisotropic functions is

$$
\begin{aligned}
\sigma\left(\widehat{u}_{1}, \widehat{u}_{2}, \widehat{r}\right) & =\sigma_{0}\left[1+\frac{1}{2}\left\{\left(l_{1}-1\right)\left|\widehat{r} \cdot \widehat{u}_{1}\right|+\left(l_{2}-1\right)\left|\widehat{r} \cdot \widehat{u}_{2}\right|\right\}\right] \\
\epsilon\left(\widehat{u}_{1}, \widehat{u}_{2}, \widehat{r}\right) & =\epsilon_{0}\left[1+\frac{1}{2}\left\{\left(d_{1}^{-1}-1\right)\left|\widehat{r} \cdot \widehat{u}_{1}\right|+\left(d_{2}^{-1}-1\right)\left|\widehat{r} \cdot \widehat{u}_{2}\right|\right\}\right]
\end{aligned}
$$


where subscripts 1 and 2 distinguish between two different types of bodies. For a complete specification of the unlike interaction, also the corresponding $\epsilon_{0}$ and $\sigma_{0}$ constants need be specified, something which can be done by the standard Lorentz-Berthelot combining rules.

Corresponding generalizations for the original Gay-Berne potential have been presented by Cleaver et al.$^{111}$ and by Berardi et al..$^{12}$ The latter of these generalizations covers also the case of biaxial molecules or molecular segments.

In closing, we make a quick comparison of the computational complexity of the modified and original potential. It is clear that eqs (2) and (3) require a much greater number of arithmetic operations than do eqs (4) and (5). Eqs (2) and (3) also require the nonelementary square-root operation. In contrast, eqs (4) and (5) require only the elementary arithmetic operations multiplication and addition. In the author's hands, the subroutine for the original potential requires approximately $15 \%$ to $25 \%$ more computer processing time (tested on two different computers). Using fast floating-point (e.g. less accurate) libraries is likely to diminish this difference somewhat. In actual simulations, the speed benefit will be somewhat less depending on system size.

\section{REFERENCES}

${ }^{1}$ J. G. Gay and B. J. Berne, J. Chem. Phys. 74, 3316 (1981).

${ }^{2}$ J. Corner, Proc. R. Soc. Lond. A 192, 275 (1948).

${ }^{3}$ T. Kihara, Revs. Modern Phys. 25, 831 (1953).

${ }^{4}$ B. C. Kohin, J. Chem. Phys. 33, 882 (1960).

${ }^{5}$ A. D. Buckingham, Adv. Chem. Phys. 12, 107 (1967).

${ }^{6}$ B. J. Berne and P. Pechukas, J. Chem. Phys. 56, 4213 (1972).

${ }^{7}$ A. Koide and T. Kihara, Chem. Phys. 5, 34 (1974).

${ }^{8}$ S. H. Walmsley, Chem. Phys. Lett. 49, 390 (1977).

${ }^{9}$ F. J. Vesely, J. Chem. Phys. 125, 214106 (2006).

${ }^{10}$ R. A. X. Persson, J. Phys. Chem. B. 115, 10073 (2011).

${ }^{11}$ D. J. Cleaver, C. M. Care, M. P. Allen, and M. P. Neal, Phys. Rev. E 54, 559 (1996).

${ }^{12}$ R. Berardi, C. Fava, and C. Zannoni, Chem. phys. lett. 236, 462 (1995). 